“C 2010 IEEE. Personal use of this material is permitted. Permission from IEEE must be obtained for all other uses, in any current or future media, including reprinting/republishing this material for advertising or promotional purposes, creating new collective works, for resale or redistribution to servers or lists, or reuse of any copyrighted component of this work in other works.” 


\title{
Research of Three-Dimensional Magnetic Reluctivity Tensor Based on Measurement of Magnetic Properties
}

\author{
Yongjian $\mathrm{Li}^{1,2}$, Qingxin Yang ${ }^{1}$, Jianguo $\mathrm{Zhu}^{2}$, Senior Member, IEEE, Youguang Guo ${ }^{2}$, Jingfeng Sun ${ }^{1}$
}

\begin{abstract}
In this paper, a method of determining the three-dimensional (3-D) reluctivity tensor by measuring 3-D vector magnetic properties is presented. Taking the Soft magnetic composite (SMC) material which is applied to 3-D magnetic flux machines as an example, this paper describes the measurement of 3-D magnetic properties and the determination of 3-D magnetic reluctivity tensor which is a second-order three-dimensional full rank matrix. Taking the off-diagonal elements into account can help to accurate numerical analysis of magnetic field in 3-D flux electrical machines.
\end{abstract}

Index Terms-anisotropy, improved 3-D tester, magnetic reluctivity tensor, soft magnetic composite (SMC)

\section{INTRODUCTION}

$\mathrm{I}^{\mathrm{n}}$ $\mathrm{N}$ magnetic field analysis, the relationship between the magnetic field strength vector $H$ and the magnetic flux density vector $B$ can be expressed by the magnetic reluctivity or permeability [1]. For ferromagnetic materials, $H$ and $B$ are hardly parallel with each other under 2-D or 3-D excitation due to the magnetic domain wall motion and rotation of local magnetization vectors [2]. The conventional magnetic field analysis using the scalar reluctivity is too simple to accurately express the phase difference between $H$ and $B$. And the corresponding magnetic properties measurement due to the Epstein tester or the single chip tester (SST) cannot express the tensor magnetic reluctivity [3]. Modeling and measurement of the magnetic properties have been done by many researchers under 2-D rotating field excitations, which are quite different from those under alternating field excitations [4]-[6]. The magnetic reluctivity becomes a tensor of full matrix in a rotating flux [5]. By using the measured 2-D data of rotating magnetic field, 2-D reluctivity tensor has been calculated and applied to 2-D finite element analysis (FEA) of the rotating magnetic field of a three-phase transformer core of grain-oriented steel [7]. In order to precisely detect magnetic field distribution of the rotating flux machine, 3-D reluctivity

\footnotetext{
Manuscript received 19 October 2009. This work is supported in part by the Science Foundation Grants of Hebei Province, China: "Three Dimensional Rotating Electromagnetic Field Numerical Analysis Based on Magnetic Reluctivity Tensor". The project number is E2008000051.

Yongjian Li. F. B. Qingxin Yang, and F. E Jingfeng Sun are with the Province-Ministry Joint Key Laboratory of Electromagnetic Field and Electrical Apparatus Reliability Hebei University of Technology, Tianjin, CO 300130 China (+862260201524; fax: ; e-mail: herolyj@hebut.edu.cn, yoli@eng.uts.edu.au).

Jianguo Zhu, Youguang Guo are with the School of Electrical, Mechanical and Mechatronic Systems University of Technology Sydney Sydney, NSW 2007, Australia. (e-mail: joe@eng.uts.edu.au).
}

tensor should be involved in electromagnetic fields analysis [8]. This paper presents the method of studying 3-D magnetic reluctivity tensor by means of 3-D magnetic properties measurement of SOMAOLY ${ }^{\mathrm{TM}} 500$, an SMC material made of highly pure iron powder with surface coating to ensure low eddy current loss [9]. The reluctivity tensor, a second-order three-dimensional full rank matrix can be derived and testified through a series of measurements on a cubic SMC sample by using an improved 3-D magnetic property tester.

\section{The IMPRoved 3-D MAgnetic Testing SysteM}

\section{A. Structure of the 3-D Tester}

A 3-D magnetic property measurement system has been successfully developed and improved in the University of Technology, Sydney (UTS) [10], [11]. The system consists of a 3 -D yoke to guide the magnetic fluxes along the orthogonal $x$-, $y$-, and $z$-axes, as shown in Fig. 1, three groups of multilayer excitation coils apply to wide range of frequency are wound around the legs of the 3-D yokes, a data acquisition system, and a feedback control system comprising three control units and three high power amplifiers.

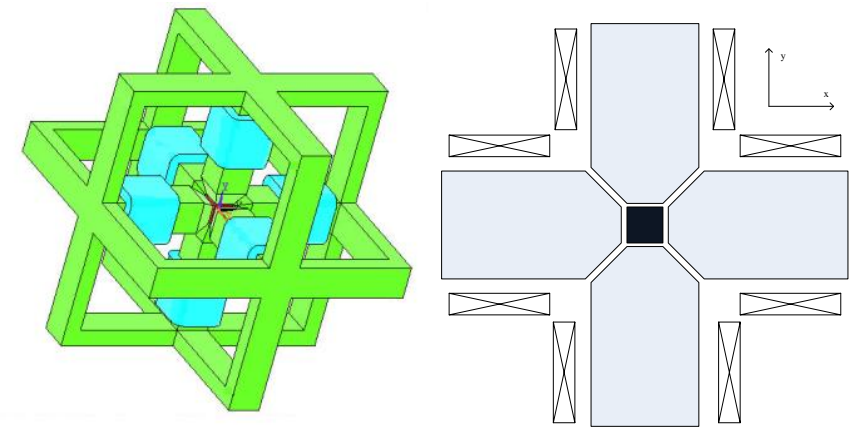

Fig. 1. Schematic diagram and cross section of the 3-D tester (skimmed the $\mathrm{z}$ direction).

\section{B. Improved Structure of the Sensing Coils}

The original sensing coils are composed by three $B$ coils wrapped around three directions of the cubic sample and $12 \mathrm{H}$ coils (six cross-type $H$ coils attached to the surface of the cubic sample) attached on the six sides of the sample [10]. The structure can be improved by considering that the surface area of the $H$ coil equals that of a side of the cubic sample and hence the magnetic field in the sensing area may not be uniform. Fig. 2 shows the improved structure of combined $H$ and $B$ coil. The thickness of the epoxy resin frame is ground to be about 0.55 $\mathrm{mm}$, and the height of the embedded $\mathrm{B}$ coil is $0.5 \mathrm{~mm}$, so the 
circle in the center of the frame can hold the $B$ coil. $H$ sensing coil is wound and attached to the surface of the frame which clings to the surface of SMC sample. So the minimized $H$ coil and the embedded $B$ coil can be attached to the central area of each side of the cubic sample. It has been found the field near the central area is quite uniform. The sensing area is now centralized and the gap between the sample and $H$ coil is contracted. This new structure of sensing coils can improve the precision of measurement. To reduce additional electromotive force (emf) in the $H$ coil, a double-cross-winding structure is adopted, as shown in Fig. 2, while two layers with a small cross angle can induce signals with equal magnitude but opposite direction, and hence cancel each other. Furthermore, the two terminals of each coil are twisted to shrink the effective area of error induction.

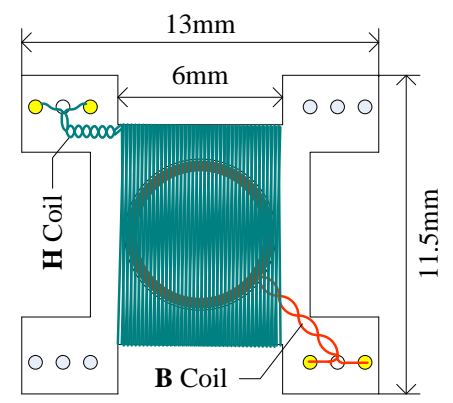

Fig. 2. Structure of combined $H$ and $B$ sensing coil

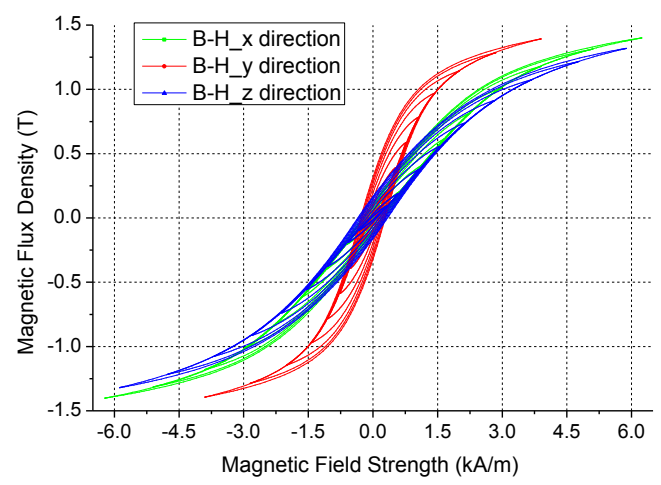

Fig. 3. Hysteresis loops for alternating magnetizations on the $x$-, $y$-, and $z$-axis.

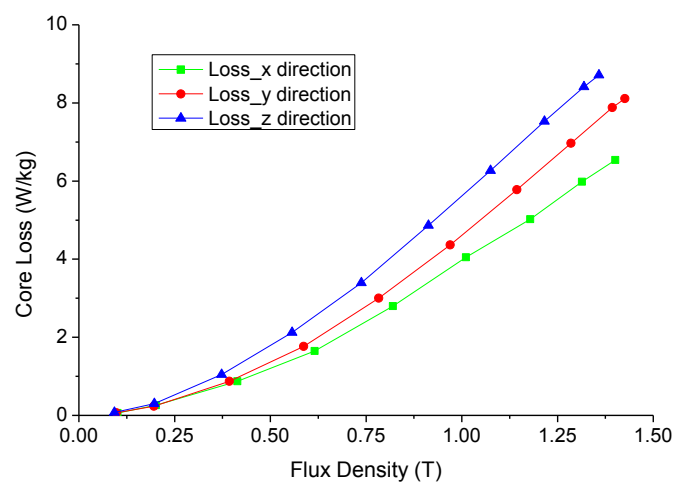

Fig. 4. Core losses for alternating magnetizations on the $x$-, $y$-, and $z$-axis.

\section{Calibration of Sensing Coils}

The calibration of the improved $H$ and $B$ sensing coils is performed in a long solenoid, which can generate a uniform magnetic field [2]. The coefficients in vertical direction of the coils are calculated by

$$
\begin{aligned}
K_{H} & =\frac{U_{H}}{\sqrt{2} \pi f \mu_{0} H_{m}} \\
K_{B_{i}} & =N_{B_{i}} A_{s p}
\end{aligned}
$$

where $U_{\mathrm{H}}$ is the $r m s$ value of the induced $e m f, \mu_{0} H_{m}$ is the peak value of the air flux density in the middle of the solenoid, $f$ is the excitation frequency, $N_{\mathrm{Bi}}$ is the number of turns of the $B_{i}$ coil, and $A_{s p}$ is the cross-sectional area of the sample. The coefficients of the other two non-effective-area directions are smaller and should be calibrated in the 3-D tester which can generate powerful field. The $H$ coil's coefficient in $z$ direction is by means of the embedded $B$ coil which is calibrated in the solenoid. Similarly, the $B$ coil's coefficient in parallel direction $K_{B}^{p} \mathrm{~K}_{\mathrm{B}}{ }^{\mathrm{p}}$ can be obtained by means of $H$ coil. The final results of calibration are obtained by averaging over a number of measurements.

When the cubic SMC sample is placed into the sensing house and subjected to a magnetic field, the voltage for the $x, y, z$ directions in H coils UH and B coils UB can be expressed as

$$
\left\{\begin{array}{l}
U_{H x}=\mu_{0}\left(K_{x}^{x} \frac{\mathrm{d} H_{x}}{\mathrm{~d} t}+K_{x}^{y} \frac{\mathrm{d} H_{y}}{\mathrm{~d} t}+\frac{K_{x}^{z}}{\mu_{0}} \frac{\mathrm{d} B_{z}}{\mathrm{~d} t}\right) \\
U_{H y}=\mu_{0}\left(\frac{K_{y}^{x}}{\mu_{0}} \frac{\mathrm{d} B_{x}}{\mathrm{~d} t}+K_{y}^{y} \frac{\mathrm{d} H_{y}}{\mathrm{~d} t}+K_{y}^{z} \frac{\mathrm{d} H_{z}}{\mathrm{~d} t}\right) \\
U_{H z}=\mu_{0}\left(K_{z}^{x} \frac{\mathrm{d} H_{x}}{\mathrm{~d} t}+\frac{K_{z}^{y}}{\mu_{0}} \frac{\mathrm{d} B_{y}}{\mathrm{~d} t}+K_{z}^{z} \frac{\mathrm{d} H_{z}}{\mathrm{~d} t}\right) \\
U_{B x}=K_{B z}^{z} \frac{\mathrm{d} B_{z}}{\mathrm{~d} t}+\mu_{0} K_{B z}^{p} \frac{\mathrm{d} H_{x}}{\mathrm{~d} t} \\
U_{B y}=K_{B x}^{x} \frac{\mathrm{d} B_{z}}{\mathrm{~d} t}+\mu_{0} K_{B x}^{p} \frac{\mathrm{d} H_{y}}{\mathrm{~d} t} \\
U_{B z}=K_{B y}^{y} \frac{\mathrm{d} B_{z}}{\mathrm{~d} t}+\mu_{0} K_{B y}^{p} \frac{\mathrm{d} H_{z}}{\mathrm{~d} t}
\end{array}\right.
$$

Then the $\mathbf{H}$ and $\mathbf{B}$ components can be worked out by solving the matrix equations.

\section{MAGNETIC PROPERTY MEASUREMENT}

\section{A. Alternating Magnetic Properties}

By using 3-D magnetic testing system, some measurements have been carried out. Fig. 3 shows the $B-H$ loops while the sample is magnetized at $50 \mathrm{~Hz}$ along the $x, y$, and $z$-axis, respectively. Fig. 4 plots the corresponding core losses. It is noticed that the loops for the $x$ and $z$-axis are similar, while the $y$-axis seems to be the easy axis, though the sample is expected to be magnetically isotropic. It means that the particles are much closer along the $\mathrm{y}$-direction than along the $\mathrm{x}$ - and z-direction, that is, high mass density and weak demagnetization field along the y-direction. It is this reason that the y-direction is an easy direction for magnetization, along which the permeability is relatively higher [11]. 


\section{B. 3-D Magnetic properties}

Fig. 5 shows a series of $\mathbf{B}, \mathbf{H}$ loci and corresponding projections when $\mathbf{B}$ loci are controlled to be circles with increasing magnitudes up to $1.4 \mathrm{~T}$ at $50 \mathrm{~Hz}$ in the $x o y, y o z$, and zox planes, respectively. It is clear that the $\mathbf{B}$ loci are well controlled to form round loci, and $\mathbf{B}$ and $\mathbf{H}$ loci lie in the same magnetization planes. On the other hand, the $\mathbf{H}$ loci in the xoy and yoz planes change from ellipse to rectangle-like with increasing magnitudes of $\mathbf{B}$ while $\mathbf{H}$ loci in the zox plane change from round to square-like shape. This phenomenon is consistent with alternating loops due to easy magnetization along y-axis.

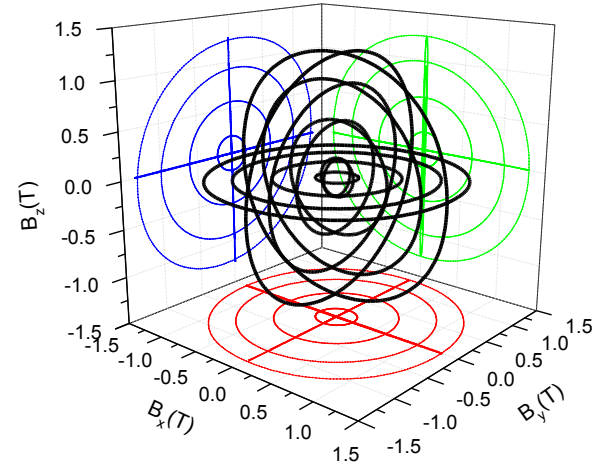

(a)

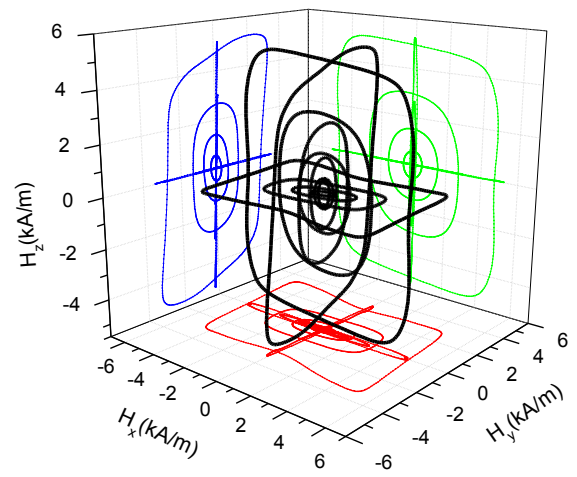

(b)

Fig. 5. Round B loci and corresponding $\mathrm{H}$ loci in xoy-, yoz, zox-plane and projections in three planes. (a) Series of B loci and corresponding projections in three planes. (b) Series of $\mathrm{H}$ loci and corresponding projections in three planes.

\section{3-D RELUCTIVITY TENSOR}

\section{A. Expression of Reluctivity Tensor}

In 1-D condition, the relationship between $B$ and $H$ can be expressed as scalar permeability or reluctivity because they are parallel. The scalar expression is

$$
B=\mu_{0} H\left(1+\chi_{m}\right)=\mu_{0} \mu_{r} H=\mu H
$$

or

$$
H=v B
$$

Where $\mu_{0}$ is free space permeability, $\mu_{\mathrm{r}}$ is relative permeability, $\chi_{m}$ is susceptibility, $v$ is reluctivity.

In 2-D or 3-D condition, the magnetic property shows nonlinearity not only in magnitude but also in the spatial phase angle between the vector $\mathbf{B}$ and $\mathbf{H}$ [12], the constitutive equation should be written as
$H_{i}=\sum_{j} v_{i j} B_{j}$

where $v_{i j}$ is the reluctivity tensor. The magnetic properties with tensor reluctivity can be called tensor magnetic properties.

According to previous 3-D magnetic property measurements and analysis, the 3-D tester can detect the real 3-D effect of tensor magnetic properties. So the $\mathbf{H}$ vector can be expressed by $\mathrm{x}, \mathrm{y}$ and $\mathrm{z}$ direction components

$$
\left\{\begin{array}{l}
H_{x}=v_{x x} B_{x}+v_{x y} B_{y}+v_{x z} B_{z} \\
H_{y}=v_{y x} B_{x}+v_{y y} B_{y}+v_{y z} B_{z} \\
H_{z}=v_{z x} B_{x}+v_{z y} B_{y}+v_{z z} B_{z}
\end{array}\right.
$$

The reluctivity tensor of the relationship between the $\mathbf{B}$ and $\mathbf{H}$ vectors can be written to a second-order three-dimensional full rank matrix

$$
v=\left[\begin{array}{lll}
v_{x x} & v_{x y} & v_{x z} \\
v_{y x} & v_{y y} & v_{y z} \\
v_{z x} & v_{z y} & v_{z z}
\end{array}\right]
$$

\section{B. Analysis of 3-D Reluctivity Tensor}

In 3-D flux rotating electrical machine, when the rotor rotates, $\mathbf{B}$ and $\mathbf{H}$ loci at different positions in the magnetic core can take very different patterns. Because any types of $\mathbf{B}$ or $\mathbf{H}$ locus can be transformed into a Fourier series, and each of the harmonics basically forms a circular or elliptical locus [13], it might be sufficient to investigate the magnetic properties of ferromagnetic materials under the excitations of elliptical $\mathbf{B}$ vectors. Maximum flux density, orientation, and axis ratio of the elliptical $\mathbf{B}$ locus are composed to define the tensor magnetic properties. The orientation is defined by the inclination angles of $\mathbf{B}$, i.e. the angles between the major axis and the three coordinate axes [14], [15].

By using measurement data Eq. (9) will be obtained, but the nine elements of reluctivity tensor cannot be solved because each element is a conditional variable. Only when the maximum flux density and axis ratio of elliptical $\mathbf{B}$ are fixed in one loop, a row of reluctivity tensor elements will be constants. If three different points of the loop are considered, a condition-specified equation set will be obtained

$$
\left\{\begin{array}{l}
H_{x}(a)=v_{x x} B_{x}(a)+v_{x y} B_{y}(a)+v_{x z} B_{z}(a) \\
H_{x}(b)=v_{x x} B_{x}(b)+v_{x y} B_{y}(b)+v_{x z} B_{z}(b) \\
H_{x}(c)=v_{x x} B_{x}(c)+v_{x y} B_{y}(c)+v_{x z} B_{z}(c)
\end{array}\right.
$$

The first row elements of reluctivity tensor $v_{x x}, v_{x y}$ and $v_{x z}$ can be acquired by solving Eq. Similarly, $v_{y x}, v_{y y}, v_{y z}, v_{z x}, v_{z y}$ and $v_{z z}$ can be obtained.

Fig. 6 illustrates the one cycle magnetic reluctivity curves in xoy plane when the $\mathbf{B}$ locus is controlled to be a circle (the ratio of elliptical locus is 1). It displays that the change of $v_{x x}$ and $v_{x y}$ is small compare with that of $v_{y y}$ and $v_{y x}$ when the magnitude of round $\mathbf{B}$ increases from $0.22 \mathrm{~T}$ to $1.32 \mathrm{~T}$ in xoy plane. It denotes that $y$-axis is the easy direction for magnetization, which is in accord with the conclusion of 
hysteresis measurement.

Fig. 7 shows the reluctivity from measured data against the magnitude of maximum round rotating $\mathbf{B}$ in a cubic SMC sample. The curves of three diagonal elements $v_{x x}, v_{y y}$ and $v_{z z}$ nearly have the same curvilinear trend when the magnitude of $\mathbf{B}$ is increasing, but the magnitude of $v_{y y}$ is smaller than that of $v_{y y}$ and $v_{z z}$. It is also consistent with the previous conclusion of easy magnetized direction. The off-diagonal elements of the 3-D reluctivity tensor $v_{x y}, v_{y z}$ and $v_{z x}$ are calculated in the boundary condition of cycle angle $\theta \approx 180^{\circ}$, and $v_{y x}, v_{z y}$ and $v_{x z}$ are calculated in the boundary condition of $\theta \approx 90^{\circ}$. The arbitrary values of these off-diagonal elements increase rapidly when the magnitude of $\mathbf{B}$ is close to saturation. As far as each off-diagonal element is concerned, the arbitrary corresponding $\mathrm{H}$ is a bigger value but the corresponding $\mathbf{B}$ is nearly zero in each location plane.

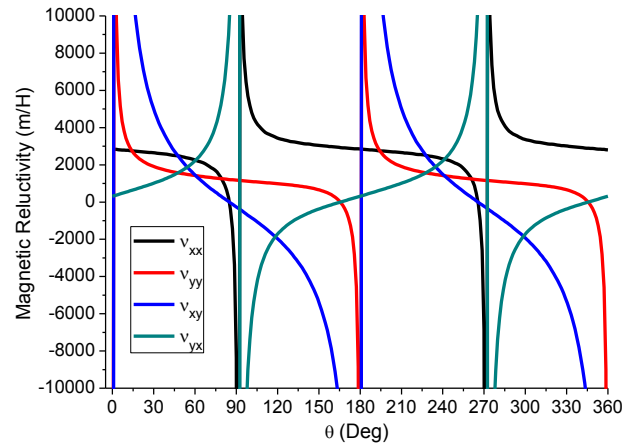

(a)

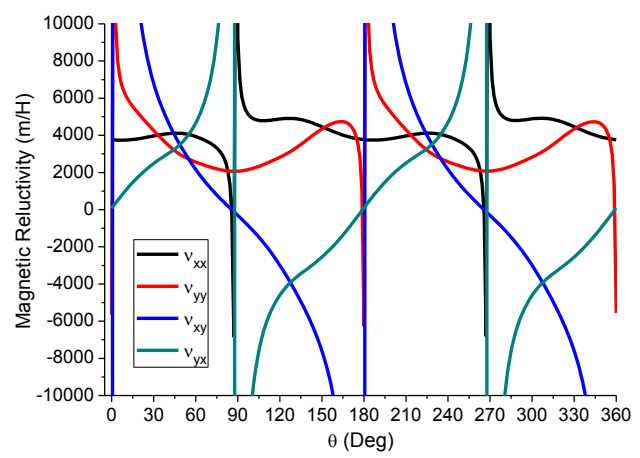

(b)

Fig. 6. Magnetic reluctivity tensor of one cycle in plane-xoy when $\mathbf{B}$ is round rotating (the axis ratio is equal to 1 ). (a) $B_{r}=0.22 \mathrm{~T}$, (b) $B_{r}=1.32 \mathrm{~T}$.

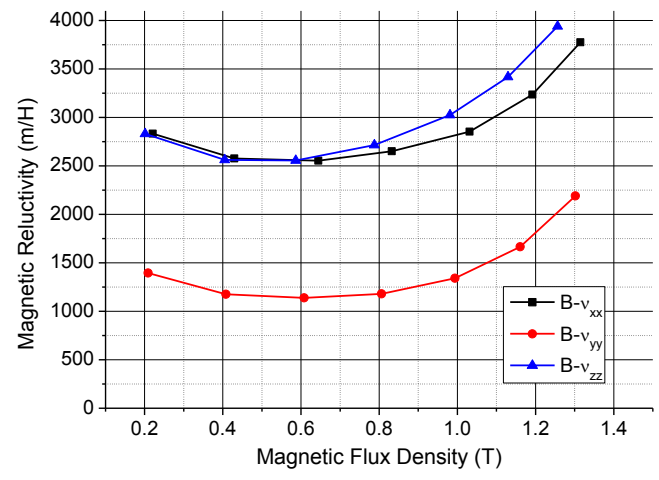

(a)

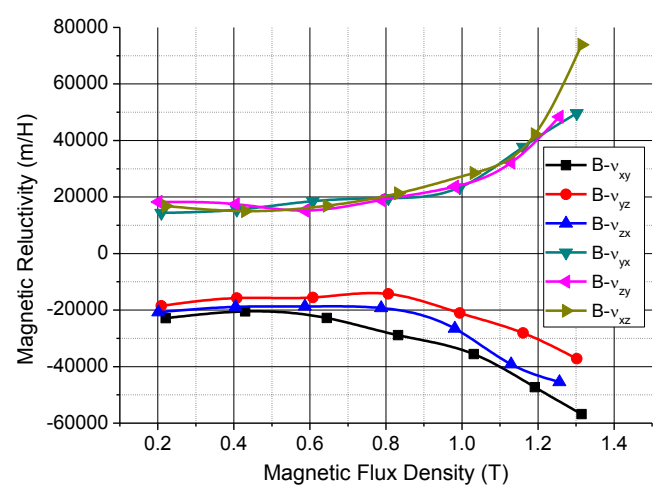

(b)

Fig. 7. Magnetic reluctivity tensor against the magnitude of the maximum round rotating B. (a) Diagonal elements, (b) Off-diagonal elements.

\section{CONCLUSION}

By using the improved 3-D magnetic tester, the 3-D magnetic reluctivity tensor of an SMC material has been analyzed by means of the measured magnetic properties. The magnetic reluctivity tensor becomes a second-order three-dimensional full rank matrix when the $\mathbf{B}$ vector is controlled to be ellipse. The off-diagonal elements of the tensor are due to the rotating magnetic excitation and are obtained under some condition of round $\mathbf{B}$ locus (ratio of the ellipse is 1). The tensor magnetic property demonstrates that the rotational magnetization is not a simple combination of individual alternating magnetization, but a complicated coupling interaction between different magnetization directions. It is helpful to quantitatively study the property of anisotropy and nonlinearity in magnetic materials at the condition of rotational magnetization. It also contributes to accurately analyze magnetic field of 3-D flux SMC machines.

\section{REFERENCES}

[1] M. Enokizono, H. Shimoji, "Magnetic characteristic analysis of electrical machines by vector magneto-hysteretic E\&S ${ }^{2}$ model," Journal of Materials Processing Technology vol. 161, pp. 218-223, 2005.

[2] V. Basso and G. Bertotti, J. Magn. Magn. Mat., 215, 1 (2000).

[3] M. Enokizono, K. Yuki, S. Kanao, "An improved magnetic field analysis in oriented steel sheet by finite element method considering tensor reluctivity," IEEE Trans. Magn., Vol. 31, No. 3, pp. 1797-1800, May 1995.

[4] J.G. Zhu, V.S. Ramsden, IEEE Trans. Magn., 29 (6) (1993) 2995.

[5] M. Enokizono, T. Todaka, and S. Kanao, "Two-dimensional magnetic properties of silicon steel subjected to a rotating field," IEEE Trans. Magn., Vol. 29, No. 6, pp. 3550-3352, Nov. 1993.

[6] J.J. Zhong, "Measurement and modeling of magnetic properties of materials with rotating fluxes," Ph.D. Thesis, Univ. Technology,Sydney, December 2002.

[7] M. Enokizono, N. Soda, IEEE Trans. Magn,. 33 (5) (1997) 4110.

[8] Y.G. Guo, J.G. Zhu, Z.W. Lin, J.J. Zhong, H.Y. Lu, and S.H. Wang, "Determination of 3-D magnetic reluctivity tensor of soft magnetic composite materials," J. Magn. Magn. Mater., vol. 312, pp. 458-463, 2007.

[9] Soft magnetic composites from Höganäs Metal Powders-SOMALOY ${ }^{\mathrm{TM}}$ 500, Höganäs Product Manual, 1997.

[10] J.G. Zhu, J.J. Zhong, Z.W. Lin, J.D. Sievert, IEEE Trans. Magn., 39 (5) (2003) 3429.

[11] Z.W. Lin and J.G. Zhu, "Three-dimensional magnetic properties of soft magnetic composite materials," J. Magn. Magn. Mater., vol. 312, pp. 158-163, 2007.

[12] M. Enokizono, H. Shimoji, A. Ikariga, S. Urata, and M. Ohoto, "Vector Magnetic Characteristic Analysis of Electrical Machines," IEEE Trans. Magn., Vol. 41, No. 5, pp. 2032-2035, May 2005. 
[13] Y.G. Guo, J.G. Zhu, Z.W. Lin, J.J. Zhong, IEEE Trans. Magn,. 41 (10) (2005) 3925.

[14] S. Urata, M. Enokizono, T. Todaka, and H. Shimoji, "Magnetic Characteristic Analysis of the Motor Considering 2-D Vector Magnetic Property," IEEE Trans. Magn., Vol. 42, No. 4, pp. 615-618, 2006.

[15] Y.G. Guo, J.G. Zhu, Z.W. Lin, J.J. Zhong, H.W. Lu, S.H. Wang, IEEE Trans. Magn. USA 42 (10) (2006) 3243. 